\title{
Vulnerabilidad social, genealogía del concepto
}

Social vulnerability, a genealogy of the concept

\author{
María Valdés Gázquez \\ Profesora Titular, Departamento de Antropología Social y Cultural, Universitat Autònoma de Barcelona (España) \\ maria.valdes@uab.cat
}

\section{CRIANZA EN CONTEXTOS DE VULNERABILIDAD SOCIOCULTURAL MONOGRÁFICO COORDINADO POR JORGE GRAU REBOLLO}

\section{RESUMEN}

El concepto de vulnerabilidad ha irrumpido con fuerza desde hace varias décadas en las ciencias sociales y, en particular, en el campo de los estudios sobre desigualdad social. Este artículo propone seguir la evolución del concepto de vulnerabilidad, concepto que surge en la década de 1970 en el intento de comprensión de desastres naturales y los problemas de recuperación de las poblaciones afectadas. De este enfoque estrictamente natural de la década de 1970 se pasó al enfoque social de la década de 1980, poniéndose el foco en el estudio de las estructuras y procesos socioeconómicos de desigualdad y pobreza como causantes de la vulnerabilidad. El boom de los estudios sobre vulnerabilidad se produce hace una década, cuando, a partir de la crisis mundial de 2008, los países desarrollados experimentaron un crecimiento de las situaciones de precariedad e inseguridad económica y social.

\section{ABSTRACT}

The concept of vulnerability has penetrated powerfully for the past few decades in Social Sciences, particularly in the field of studies in social inequality. In this article, we intend to follow the evolution of the concept of vulnerability, a concept that emerged in the 1970s and was used to comprehend natural disasters and the recovery problems of affected populations. After the strictly natural approach of the 1970s, the social approach emerged in the 1980s, focusing on the study of socioeconomic structures and processes of inequality and poverty as causes of vulnerability. The boom in vulnerability studies occurred a decade ago, when, after the world crisis of 2007-2008, developed countries experienced an increment of precarious situations and economic and social insecurity.

PALABRAS CLAVE

vulnerabilidad social | desigualdad | riesgo | movilidad social | pobreza

KEYWORDS

social vulnerability | inequality | risk | social mobility | poverty

\section{Introducción}

El concepto de vulnerabilidad ha irrumpido con fuerza desde hace varias décadas en las Ciencias Sociales y, en particular, en el campo de los estudios sobre desigualdad social. Se ha convertido en una herramienta analítica utilizada en el estudio de la realidad social, especialmente en los informes elaborados por organismos internacionales (Naciones Unidas, OIT, OCDE, el Banco Mundial) para diseñar y orientar las políticas públicas en materia de desarrollo socioeconómico, así como las intervenciones de acción humanitaria.

En este artículo nos proponemos trazar la genealogía del concepto de vulnerabilidad social, rastrear su origen y su desarrollo, describir cómo se ha ido trasladando de unas disciplinas a otras, de un conjunto de textos a otros y dar las claves de por qué determinada acepción del concepto ha tenido un éxito particular en un momento y lugar concretos. Todo ello con el objeto de calibrar la carga histórica del concepto y su posible influencia en la potencialidad de su uso en la investigación social.

Como muchos de los conceptos manejados en ciencias sociales, "vulnerabilidad" es un término del lenguaje común que, una vez se traslada a un uso científico, lleva consigo el lastre de su significado 
ordinario. Por ello, no está de más comenzar con definiciones de diccionario. El de la RAE define "vulnerabilidad" como "cualidad de vulnerable" y la definición de "vulnerable" es "que puede ser herido o recibir lesión, física o moralmente". El origen etimológico del término vulnerabilidad (Feito 2007: 9) nos remite a términos latinos como vulnus (herida, golpe, punzada, y también desgracia o aflicción), vulneratio (herida o lesión), o vulnero (herir o lastimar). Si éstos apuntan a una amenaza exterior, el término castellano se refiere, en cambio, a una cualidad de fragilidad con respecto a esa amenaza. Esas dos vertientes del significado del término en su uso común se han trasladado a su uso científico, que toma en consideración el riesgo, las amenazas, las crisis que pueden infringir daño y también la fragilidad, condición que compartimos todos los organismos vivos y que nos hace susceptibles de ser dañados.

En lo que sigue me centraré en el origen y el desarrollo del uso científico del concepto, pasando del enfoque natural de la década de 1970 al enfoque social de la década de 1980; trataré de caracterizar los enfoques teóricos de la vulnerabilidad social, me detendré especialmente en el éxito particular que tuvo este concepto en Latinoamérica en torno al año 2000 y concluiré con las principales críticas que ha recibido.

\section{Del enfoque natural al enfoque social: de los desastres naturales a la pobreza}

Pérez de Armiño (2000) ha situado la aparición del concepto en los textos científicos en la década de 19 70 , con el intento de comprensión de ciertos desastres naturales (sequías, huracanes y terremotos) y los problemas de recuperación de las poblaciones afectadas. Los desastres se contemplaban como eventos excepcionales, inesperados, consecuencia directa de factores naturales imprevistos (meteorológicos, medioambientales o demográficos), sin interrogarse especialmente por la posible interrelación con factores sociales. Como mucho, se valoraba la interacción entre el desastre natural y la mala gestión de los recursos naturales por parte de las víctimas (sobrecultivo, sobrepastoreo, tala abusiva de los bosques, etc.).

Según el mismo autor, de este enfoque estrictamente natural de la década de 1970 se pasó, en la década de 1980, a un enfoque social. Este enfoque acepta la importancia de las catástrofes naturales como eventuales activadores de los desastres, pero pone el foco en el estudio de "las estructuras y procesos socioeconómicos de desigualdad y pobreza como causantes de la vulnerabilidad" (Pérez de Armiño 2000: 2). De esa forma, una vez se introdujeron los factores sociales en la comprensión de los desastres, el concepto de vulnerabilidad no se empleó únicamente para estudiar la reacción de la población ante los desastres naturales exógenos, sino también para comprender en qué medida las estructuras sociales sitúan a algunos sectores de la población en la indefensión.

Los orígenes del concepto están de esa manera muy marcados por los programas de acción humanitaria y de cooperación al desarrollo, por las relaciones que se establecieron entre los países del primer mundo, proveedores de ayuda, y los países en vías de desarrollo. Una contribución esencial en la conformación del concepto fue la del economista indio Amartya Sen, quien en Poverty and Famines (1981) analizó las hambrunas desde la perspectiva conceptual de las titularidades (entitlements) sobre el alimento y puso el foco en la pobreza y la desigualdad social. Sen explicaba las hambrunas como consecuencia, no de la escasez de alimentos provocada por una catástrofe o por el exceso de población, sino como una dificultad de acceso a los mismos por parte de las familias con menos recursos económicos: "El hambre caracteriza a personas que no tienen suficiente alimento para comer, y no a una situación en la que no hay suficiente alimento disponible" (1):

Desde ese trabajo seminal de Amartya Sen el concepto fue penetrando en los análisis del desarrollo socioeconómico realizados desde diversas disciplinas y sufragadas por diversos organismos internacionales (2). El boom de los estudios sobre vulnerabilidad, a juzgar por las fechas de publicación de las contribuciones esenciales, se produce en la primera década del siglo XXI. Es en esas fechas cuando el concepto de vulnerabilidad se convierte en una herramienta esencial para diseñar y orientar 
las políticas públicas en materia de desarrollo, así como las intervenciones de acción humanitaria.

El porqué lo ha señalado muy bien Nicolas Sirven (2007) (3): en el año 2000 se establecieron los Objetivos de Desarrollo del Milenio, también conocidos como Objetivos del Milenio (ODM), ocho propósitos de desarrollo humano que los 189 países miembros de las Naciones Unidas acordaron conseguir para el año 2015. El primero de dichos Objetivos es precisamente el de "erradicar la pobreza extrema y el hambre, acrecentar las condiciones de vida sobre la tierra en un nivel aceptable para todos". A la vez, en esas mismas fechas, y sobre todo a raíz de la crisis mundial de 2008, los países desarrollados han experimentado un crecimiento de situaciones de precariedad e inseguridad económica y social.

No es de extrañar que un buen contingente de especialistas de diversas disciplinas -sobre todo, pero no solo, del ámbito de las ciencias sociales- centrase sus esfuerzos en el análisis de la pobreza. Sirven asegura que se ha producido una evolución conceptual y metodológica en esos análisis de la pobreza hasta concebir los útiles susceptibles de anticipar la pobreza: uno de los principales sería la noción de vulnerabilidad.

"Pobreza" sería un concepto ex post, mientras que "vulnerabilidad" es un concepto ex ante. La pobreza sería una medida de un estatus actual; la vulnerabilidad, en cambio, tiene cierta cualidad predictiva:

"Il s'agit là d'une dimension nouvelle dans la lutte contre la pauvreté: les stratégies reposaient jusqu'à présent sur une analyse ex-post des situations; désormais, il faut anticiper les trajectoires de la pauvreté, c'est-à-dire adopter une vision ex-ante. C'est dans ce cadre que l'analyse de la pauvreté s'est orientée vers celle de la vulnérabilité" (Sirven 2007: 9).

Ese margen de predictibilidad es el que ha convertido el concepto de vulnerabilidad en un conceptofetiche en el ámbito de las políticas públicas. Si el traspaso del umbral de la pobreza, la disminución del bienestar de una familia por debajo de ese umbral, es predecible, entonces las políticas públicas deben actuar para detectarlo, para evitarlo antes de que se produzca y para paliarlo en caso de que se produzca.

\section{Enfoques teóricos de la vulnerabilidad social: riesgo y fragilidad}

La irrupción del concepto de vulnerabilidad alrededor del año 2000 en el campo de los estudios sobre desigualdad social y pobreza fue rotunda, de manera que se ha convertido en un concepto central en muchos de los estudios sociales sobre esos tópicos desde entonces. La noción de desigualdad social es demasiado abstracta, demasiado inespecífica y hasta cierto punto, en la medida en que depende de un juicio ético, arbitraria. Ese sustrato inespecífico ha dado al traste con la elaboración de propuestas de sistematización conceptual que dotasen de herramientas analíticas aplicables a la realidad social. Los textos sobre desigualdad social, pese a ser reveladores, tienen un carácter más teórico, incluso filosófico, pero son de difícil aplicación.

La noción de vulnerabilidad social se ha revelado más terrenal, más operacionalizable, más medible. EI concepto se ha utilizado como enfoque central en numerosos estudios empíricos y buena parte de los estudios de carácter teórico básicamente de lo que se ocupan es del desarrollo del entramado conceptual que se precisa para completar el análisis de los casos estudiados. La cantidad de textos publicados tomando como eje central la noción de vulnerabilidad social es ingente, pero dado que nuestro interés se centra en ofrecer las claves históricas de su desarrollo, apuntaremos a una clasificación que indudablemente deja textos fuera, pero arroja mucha luz sobre el contexto propicio para su formulación.

Nos referimos a la distinción que establece Moreno Crossley (2008) entre dos enfoques teóricos de la vulnerabilidad social que se corresponden con dos interpretaciones características de su significado (coincidentes con los dos significados que antes distinguimos en su uso común): vulnerabilidad y riesgo, 
vulnerabilidad y fragilidad (4). La primera concepción, sostenida principalmente por autores europeos, introduce la noción de riesgo como elemento constitutivo esencial de la vulnerabilidad social; la segunda, defendida por autores latinoamericanos, vincula la vulnerabilidad social a la vivencia de situaciones de fragilidad, precariedad, indefensión o incertidumbre. No son mutuamente excluyentes, sino en muchos casos concomitantes, pero lo que aquí nos interesa es caracterizarlas y vincularlas con el contexto de su formulación.

\section{Vulnerabilidad y riesgo}

En el marco de la discusión teórica en Ciencias Sociales se produjeron a mediados de la década de los 80 un conjunto de conceptualizaciones vinculadas a la noción de riesgo con el objetivo de redefinir su contenido como herramienta conceptual y analítica en la investigación social:

"En el núcleo de esta nueva generación de definiciones encontramos la tendencia a tematizar el riesgo como una fuerza en estado potencial que -a la manera de reverso de los procesos de modernización y racionalización característicos del proyecto histórico de la modernidad- supone la producción colateral de amenazas y -eventualmente- de daños que entrañan peligro para la adaptación y la propia existencia de los individuos y de los colectivos humanos en el planeta" (Moreno 2008: 17).

En los análisis de la vulnerabilidad social se identifica el riesgo con la noción de contingencia, un factor potencial de generación de perjuicios que pueden afectar a individuos o colectivos humanos. De esa manera, es posible concebir un conjunto de situaciones de riesgo que pueden tener o no, dependiendo de los cursos de acción, consecuencias negativas para los sujetos socializados. Con esta concepción, el riesgo puede operacionalizarse en términos de probabilidades. Hay situaciones que implican más riesgo que otras y, del mismo modo, también hay determinados individuos o poblaciones que, dependiendo de sus características, van a estar más expuestos a sufrir perjuicios en el caso de producirse determinadas situaciones.

Nos detendremos aquí en las definiciones del riesgo que Moreno Crossley (므) considera característicamente sociológicas. Se trata de definiciones que integran el concepto de vulnerabilidad social en el contexto de la discusión teórica sobre la crisis del proyecto de la modernidad occidental. Los principales representantes de este tipo de análisis son autores europeos que han teorizado sobre la sociedad del riesgo: Niklas Luhmann, Ulrich Beck, Anthony Giddens y Gosta Esping-Andersen. El riesgo sería un correlato de los sistemas sociales del capitalismo avanzado que trastocaría de manera inevitable los procesos de modernización marcando un punto de inflexión en el proyecto de la modernidad occidental, representando una amenaza sustantiva para sus propias bases.

Ulrich Beck (1986) es quien ha puesto más empeño en caracterizar la sociedad del riesgo, producida por una mutación del modelo característico de la sociedad industrial, a partir de efectos no deseados del proceso modernizador que escapan del control de las instituciones y se convierten en una seria amenaza a sus premisas. Se trata de una amenaza de carácter irreversible y crecientemente global que no solo atañe a la vida de los seres humanos, sino también a la de las plantas y los animales de nuestro planeta. El riesgo, así concebido, no se relaciona únicamente con un sector de la sociedad, con situaciones de pobreza o escasez material, sino que representa una amenaza al conjunto de la sociedad: los riesgos acaban por afectar a quienes los producen para obtener algún beneficio. Según Beck, aunque todavía está vigente la lógica que gobernó las anteriores etapas del proceso de modernización (la lógica de producción y reparto de riquezas cuya matriz es la desigualdad de clases sociales), en esta nueva etapa histórica ha aparecido una nueva lógica, la de producción de riesgos, que dará origen a nuevas formas de fragmentación y desigualdad social y acabará convirtiéndose en el principio rector del modelo de sociedad que está emergiendo en los países de Europa occidental.

Coincidimos con Moreno Crossley en que esta teorización que vincula riesgo y vulnerabilidad procede de "una mirada particularmente condicionada por la realidad de las sociedades europeas contemporáneas, en especial aquellas situadas en una fase de declive de los modelos de integración social del tipo Estado 
benefactor o de bienestar (Welfare State)" (Moreno 2008: 18). Estos autores (Beck es una muestra) ponen mucho empeño en proyectar la idea del riesgo en una perspectiva histórica que engarza con los problemas sociopolíticos de los países de Europa occidental de finales de la década de 1990.

\section{Vulnerabilidad y fragilidad}

Las concepciones que vinculan vulnerabilidad y fragilidad se centran en los procesos de exclusión social: cualquier unidad de análisis juzgada como "vulnerable" se encuentra en una posición de desventaja con respecto al resto y, dadas sus condiciones, su situación es susceptible de empeorar en un futuro próximo. Dicha noción de vulnerabilidad se incardina en el estudio sistemático de las diversas formas de desigualdad social y ha sido empleada especialmente por autores latinoamericanos en el análisis socioeconómico de sus sociedades.

Lo más característico de estas concepciones es que se centran en el estudio de unas formas específicas de desigualdad, las desigualdades dinámicas, definidas por oposición a la noción de desigualdades estructurales. Las desigualdades estructurales son sistémicas: el sistema favorece la diferenciación de una serie de grupos o categorías a partir de su competencia por los recursos. En las sociedades de mercado capitalistas, las desigualdades estructurales del sistema de clases plasmadas en la jerarquía socio-ocupacional son legitimadas por el modelo meritocrático y se presentan como diferencias funcionales del sistema. Las desigualdades dinámicas remiten a un conjunto de diferencias sociales que escapan a esta lógica:

"Estas son -en rigor- desigualdades observadas dentro de categorías o de grupos sociales más amplios, que cobran expresión en la coexistencia de diversos niveles de bienestar o en las diferentes expectativas u oportunidades de vida que se observan entre individuos que poseen una dotación de atributos o una trayectoria relativamente similares. Las desigualdades dinámicas son aceptadas mientras posean un carácter transitorio, pero tienden a hacerse poco tolerables en la medida en que se estabilizan en el tiempo" (Moreno 2008: 14).

Esa persistencia en el tiempo de situaciones de inestabilidad e incertidumbre que afectan a individuos o poblaciones y que los diferencian de otras categorías sociales por la vía de la exclusión social es precisamente aquello de lo que pretenden dar cuenta los análisis a los que nos estamos refiriendo. Ejemplos de dichos análisis los constituyen los trabajos realizados por autores latinoamericanos, como Rubén Kaztman o Carlos Filgueira, para agencias regionales de la Organización de las Naciones Unidas tales como la Organización Internacional del Trabajo (OIT) y la Comisión Económica para América Latina y el Caribe (CEPAL).

También en este caso nos encontramos con que esta teorización que vincula vulnerabilidad y fragilidad procede de una mirada muy condicionada por la situación de los países latinoamericanos alrededor de la década del 2000: la inestabilidad económica propiciada por la globalización, la crisis del empleo formal y el incremento de la pobreza con "la irrupción de los llamados 'nuevos pobres', los segmentos de clase media que, a pesar de su capital educativo y los esfuerzos por insertarse en el mercado laboral, quedaron al margen de la distribución de los recursos societales y experimentaron la ruptura del ideal meritocrático" (González 2009: 13).

A continuación haremos una incursión con un poco más de detalle en una de las teorizaciones más fructíferas de esta concepción, la del enfoque AVEO formulado por un grupo de autores latinoamericanos que realizaron numerosos estudios financiados por la OIT y la CEPAL durante la primera década del siglo XXI. Lo hacemos con dos intenciones: la de ofrecer una caracterización más completa de una propuesta con un notable desarrollo conceptual y la de tratar de mostrar la razón por la cual el enfoque de la vulnerabilidad social tuvo un éxito especial en América Latina. 
Se trata de un enfoque que tiene su inspiración original en el análisis de Amartya Sen (1981) basado en las desigualdades sociales y la pobreza y, más concretamente, en el enfoque basado en activos de Caroline Moser, inspirado en aquél. En 1998, Caroline Moser, una antropóloga social urbana que, becada por la fundación Ford y con financiación del Banco Mundial, había realizado numerosos trabajos empíricos sobre pobreza urbana en Ecuador, Colombia y Guatemala, publicó un artículo en el que presentó su enfoque basado en activos: "Reassessing urban poverty reduction strategies: The asset vulnerability framework". Su análisis supuso un cambio significativo de registro en los estudios sobre pobreza: propuso prestar más atención a aquello que los pobres tienen que a aquello de lo que carecen; Moser llegó a hablar de "portafolios de activos" (portafolios en el sentido de mochila). Son dos los conceptos básicos en su análisis:

1) Vulnerabilidad social: el conjunto de limitaciones o desventajas que las personas encuentran para acceder y usar los activos que se distribuyen en la sociedad.

2) Activos: el conjunto de bienes, recursos o atributos (materiales o intangibles) que pueden ser administrados para mejorar el nivel de bienestar o superar situaciones adversas.

El enfoque basado en activos de Caroline Moser fue adaptado a la realidad latinoamericana por Rubén Kaztman y Carlos Filgueira, consultores de desarrollo económico y miembros de la oficina de la CEPAL en Montevideo e investigadores de la Universidad Católica de la misma ciudad (므):

Aunque el punto de partida de estos autores es el "asset vulnerability approach" de Caroline Moser, introducen una corrección sustancial. Están de acuerdo con Moser en que las políticas para la reducción de la pobreza deben tener en cuenta la configuración de activos de las familias y sus formas de uso y reproducción. Coinciden, asimismo, con Moser en que no podemos preguntarnos "qué podemos hacer por los pobres" sin considerar "qué hacen los pobres por ellos mismos", sin contar con los activos que manejan, con los recursos de que disponen los hogares y con las formas en que son usados. Este cambio de registro en el abordaje de la pobreza es asumido por el enfoque AVEO, pero aquí plantean una corrección. Los recursos de los hogares y las formas en que son usados dependen de los esfuerzos propios, pero también dependen de algo más: de las estructuras de oportunidades provistas por el Estado, el mercado y la sociedad, es decir, dependen de los cambios en el mercado, de las prestaciones estatales y del acceso a recursos comunitarios, todos ellos variables.

Por ello, proponen pasar de la pregunta central del enfoque moseriano (¿qué recursos tienen los hogares y cómo los movilizan para enfrentar situaciones de vulnerabilidad?) a una pregunta modificada que incorpora la noción de estructura de oportunidades: ¿qué recursos poseen los hogares y qué estructura de oportunidades ofrecen el mercado, el Estado y la sociedad para escapar a situaciones de pobreza $y$ también de vulnerabilidad? (Kaztman y otros 1999: 34).

Si el planteamiento de Moser se puede resumir en activos y vulnerabilidad (AV), el de estos autores añade un elemento más: activos, vulnerabilidad y estructura de oportunidades (AVEO). Este nuevo enfoque tiene, según los autores, dos virtudes: "a la posibilidad de articular el análisis micro de los hogares (activos) con el análisis macro de las tres instituciones básicas del orden social (esto es, de las estructuras de oportunidades provistas por el Estado, el mercado y la sociedad), y permite investigar problemas de vulnerabilidad en categorías sociales distribuidas a todo lo largo del sistema de estratificación" (Kaztman y otros 1999: 93).

A partir de esta premisa construyen todo un entramado conceptual centrado en la noción de vulnerabilidad social, que conciben como una relación entre dos términos: por una parte, la estructura de oportunidades y, por otra, las capacidades de los hogares(Kaztman y otros 1999: 166). En el siguiente diagrama, de elaboración propia, hemos tratado de representar el desarrollo conceptual (vulnerabilidad social, estructura de oportunidades, capacidades, recursos, activos y pasivos) y la teorización de la vulnerabilidad social del enfoque AVEO: 


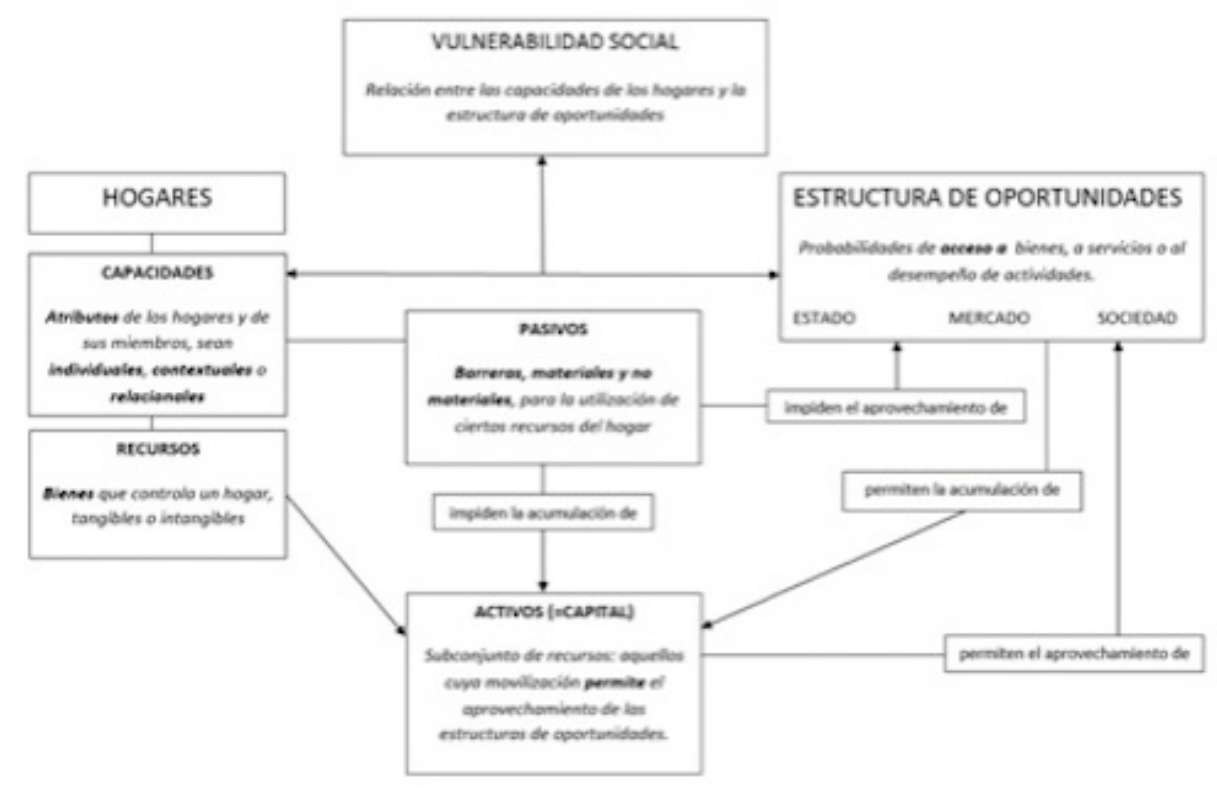

Según sus autores, la principal virtud de este enfoque es que su carácter dinámico permite analizar diferentes realidades: obliga a preguntarse por los patrones efectivos de movilidad social y de integración social en cada momento dado. Los recursos que manejan los hogares se definen como activos en función de su utilidad para aprovechar la estructura de oportunidades que se presenta en un momento histórico y en un lugar determinados. De esa forma, no tiene sentido hablar de activos fuera del contexto de los patrones de movilidad e integración social y de las estructuras de oportunidades que los acompañan. Como esos patrones y esas estructuras se transforman continuamente con el desarrollo y el progreso técnico, ciertos recursos de los hogares pierden su carácter de activos y otros lo ganan (Kaztman y otros 1999: 35).

Pero ¿por qué este tipo de análisis centrados en la vulnerabilidad social eran especialmente aptos para el análisis de la realidad socioeconómica latinoamericana? ¿Por qué el concepto de vulnerabilidad social tuvo un éxito tan espectacular en América Latina? (7). Filgueira se lamentaba de que en el caso de América Latina los estudios previos sobre estratificación social se habían focalizado casi exclusivamente en los fenómenos de la pobreza y la exclusión social; según él, en América Latina "conocemos mucho más sobre los pobres, los indigentes y los marginales que sobre las condiciones de vida, alineamientos sociales y movilidad de las clases bajas urbanas integradas o de las “clases medias'” (Filgueira 2001: 8).

Especialmente interesante es la defensa que Filgueira (2001: 9) hace de las bondades del enfoque de vulnerabilidad social, básicamente porque rompe con la dicotomía pobre/no pobre y se centra en el fenómeno del bienestar social desde una perspectiva dinámica:

"hace su aporte en tanto escapa a la dicotomía pobre/no pobre, proponiendo la idea de configuraciones vulnerables (susceptibles de movilidad social descendente, o poco proclives a mejorar su condición), las cuales pueden encontrarse en sectores pobres y no pobres. La madre sotera, el trabajador cuya calificación se ha hecho obsoleta, parejas en ciertas etapas del ciclo vital, el joven que no estudia ni trabaja, son tan solo los ejemplos más gruesos de una conceptualización que observa el fenómeno del bienestar social desde una perspectiva intrínsecamente dinámica".

No se trata únicamente de un concepto, sino de un enfoque: los estudios de vulnerabilidad social ponen el foco en las "oscilaciones de la movilidad social de comunidades, hogares y personas" (González 2008: 11). Ofrecen una mirada complementaria, un enfoque (en el sentido de poner el foco) complementario, a los estudios que se centran en las situaciones de clara desventaja social, como la pobreza y la exclusión. En realidad, los estudios de vulnerabilidad social se centran fundamentalmente en las clases medias, en los sectores que pueden traspasar con cierta facilidad el umbral de la pobreza, están muy directamente 
relacionados con las políticas públicas, porque se orientan a la detección de fragilidades y a la prevención de esa caída social.

No es de extrañar que, como efecto de la crisis económica de 2008, hayan proliferado aún más los estudios sobre vulnerabilidad (ㅇ), dado que esa crisis ha supuesto un incremento sustantivo de situaciones de fragilidad entre la población en prácticamente todos los países, incluso en economías robustas en las que cabía suponer la existencia de una clase media consolidada. Ello ha producido una nueva generación de textos que emplean como concepto central el de vulnerabilidad social. La pandemia que nos ha golpeado en 2020 , cuando se empezaban a apreciar señales de recuperación de la crisis, incrementará con toda seguridad este tipo de aproximaciones, de manera que hemos de ser muy conscientes de algunas de las debilidades que se han señalado en el concepto.

\section{Críticas al concepto de vulnerabilidad social}

Pese al éxito considerable del que ha gozado el concepto de vulnerabilidad social en las dos últimas décadas, no obstante la proliferación de investigaciones que lo ponen en el centro de su enfoque, también ha sido objeto de críticas entre algunos círculos de investigadores que lo han excluido de su uso. Es importante señalar que esas críticas no se dirigen a uno u otro de los enfoques teóricos de la vulnerabilidad, sino que son críticas al propio concepto. Nos centraremos en las tres críticas principales que ha distinguido Moreno Crossley y que pueden resumirse en una: la sospecha sobre el anclaje ideológico del concepto.

La primera crítica hace referencia a la tendencia de los enfoques de vulnerabilidad a la individualización de las causas de la desigualdad. Los análisis fragmentan en demasía las condiciones de desigualdad, los grupos o sujetos considerados vulnerables lo son por la confluencia de formas localizadas de privación y exclusión social y por su incapacidad de hacerles frente y adaptarse a ellas. De esa manera, se subraya lo singular y lo específico de cada situación de vulnerabilidad social y, a la vez, se concede demasiada importancia a la acción individual, omitiendo el rol de la acción colectiva en la lucha frente a la desigualdad social:

"Este sesgo revela una manifiesta omisión del rol de la acción colectiva y de la influencia que procesos de mayor dimensión y complejidad, como lo son las dinámicas de la acumulación capitalista, o de la evolución histórica de formas extensivas de exclusión social relacionadas con sistemas categoriales de desigualdad (que producen discriminaciones de crucial importancia como las de sexo-género, étnicas, de ciudadanía, etc.)" (Moreno 2008: 29-30).

La segunda crítica incide en la facilidad con la que se ajusta el concepto de vulnerabilidad social al paradigma neoliberal de interpretación de la desigualdad, algo que se evidencia claramente en la pregunta central de quienes lo usan por la "movilidad social" (que no por la "movilización social"). No es difícil, en efecto, apreciar en estos enfoques la impronta de las concepciones neoliberales. En el caso del enfoque AVEO en el que nos hemos detenido, las desigualdades dinámicas en las que se concentran estos autores designan un conjunto de diferencias sociales que, según presumen, escapan a la lógica de las desigualdades estructurales (estratos o jerarquías de ingresos asociadas a diferentes estratos socioocupacionales dentro de una economía de mercado). Como bien señala Moreno Crossley (2008: 30), esta visión se corresponde "con un razonamiento que observa la descomposición de las formas de desigualdad clasista como un proceso que abre las puertas al incremento de movilidad social individual y a una reducción significativa de las diferenciales oportunidades de vida entre miembros de distintas clases sociales". Una desigualdad dinámica es más fácilmente tolerable que una desigualdad estructural. Abordar el fenómeno de la desigualdad a través de la noción de desigualdad dinámica implica una cierta confianza en el sistema, que permitiría el ascenso social.

La tercera crítica al concepto de vulnerabilidad social tiene que ver con el papel que ha jugado en el diseño e implementación de programas sociales de gobierno, orientados a ayudar a los más vulnerables 
o a aquellos que se encuentran en riesgo social. La aplicación de criterios de vulnerabilidad o de riesgo social ha imprimido formas marcadamente asistencialistas de hacer política social. El problema se produce cuando tienen que establecerse criterios para designar a los beneficiarios de esas políticas asistenciales, criterios que suelen basarse en indicadores de vulnerabilidad que muchas veces dejan fuera a personas o grupos que pueden necesitarlas de la misma forma. Nos son muy conocidos ejemplos de nuestro propio país: las disfunciones de la aplicación de la ley de la dependencia o de la ley del ingreso mínimo vital.

\section{Conclusiones}

Concluimos esta exploración de la genealogía del concepto "vulnerabilidad social" resumiendo algunos de los elementos clave que conforman lo que hemos denominado la "carga histórica" del concepto, lo que debería permitirnos calibrar el potencial de su uso en la investigación social, pero también algunas prevenciones sobre ese uso.

El concepto comienza a usarse en la década de 1970 para dar cuenta de los problemas de recuperación de las poblaciones afectadas por ciertos desastres naturales, de manera que los orígenes del mismo están muy marcados por los programas de acción humanitaria y de cooperación al desarrollo, por las relaciones que se establecieron entre los países del primer mundo, proveedores de ayuda, y los países en vías de desarrollo.

En las ciencias sociales el concepto de vulnerabilidad se introdujo a partir de la década de 1980 en los estudios sobre desigualdad social y pobreza, con cierta ventaja por lo que se refiere a aplicabilidad sobre estos dos conceptos, con los que comparte campo semántico. La noción de desigualdad social es demasiado abstracta, la noción de vulnerabilidad social es más operacionalizable, más medible. El concepto de pobreza es un concepto ex post, el de vulnerabilidad es un concepto ex ante. Esas son las razones por las que el concepto de vulnerabilidad se ha convertido en un concepto-fetiche en el ámbito de las políticas públicas y aparece no solo en los textos científicos sino también en el discurso político y mediático.

Los enfoques del concepto de vulnerabilidad social de la década de 1990 y de la década del 2000 están muy condicionados por la situación de los países en los que se produjeron. Las teorizaciones de los autores europeos en la década de 1990 responden a la realidad de los problemas sociopolíticos de los países de Europa occidental derivados del declive del Estado del bienestar. Las teorizaciones de los autores latinoamericanos alrededor de la década del 2000 se vinculan con los problemas de sus países, en particular la irrupción de los "nuevos pobres" derivada de la crisis del empleo formal y el incremento de la pobreza como efecto de la globalización. La crisis de 2008 supuso el incremento sustantivo de situaciones de fragilidad entre la población en prácticamente todos los países, con la consecuente proliferación de estudios sobre vulnerabilidad. La pandemia de la Covid-19 de 2020 con toda probabilidad tendrá un efecto multiplicador de este tipo de estudios.

Aviso para navegantes: quienes adopten este enfoque seducidos por la potencialidad del concepto deberán ser muy conscientes de su carga histórica que, en buena medida, está en el trasfondo de la carga ideológica por la que lo han criticado y desechado algunos círculos de investigadores.

\section{Notas}

Esta publicación es resultado de la investigación que he llevado a cabo en el proyecto de I+D Crianza, desamparo y vulnerabilidad sociocultural. Análisis situacional y propuestas de intervención (CSO2017-83101-C2-1-R). 
1. Abriendo el nuevo milenio, el historiador y sociólogo urbano estadounidense Mike Davis (2001) vino a completar de forma asombrosa, dándole un giro, la perspectiva de Sen y sus seguidores mediante una poderosa investigación acerca de los efectos causados por la aceleración de los procesos colonizadores en la segunda mitad del siglo XIX Como revela Davis, este período presenció un verdadero "holocausto", con un saldo de entre 30 y 50 millones de muertos, a raíz de una serie de fenómenos "naturales" (sequías, epidemias y otros desastres) en un área extensísima del mundo (subcontinente indio, China, Brasil y otras muchas zonas). Las descripciones del autor son sobrecogedoras (desde las muertes masivas por inanición hasta la práctica generalizada del canibalismo para poder sobrevivir, pasando por la proliferación de plagas de insectos o la venta de los propios hijos, etc.), pero lo que, a los efectos del presente artículo, resulta interesante, tanto como inquietante y elocuente, es que estos fenómenos se habían dado durante siglos en esas mismas zonas, en relación sobre todo con el fenómeno climático conocido como El Niño, pero nunca en proporciones tan gigantescas como las que adquirieron en la era victoriana tardía. La tesis de Davis es que las potencias que competían por el reparto del mundo por aquel entonces no sólo aprovecharon catástrofes como El Niño para apresurar su toma de posesión, sino que lo hicieron a costa del desmantelamiento, asimismo apresurado pero eficaz a la postre, de instituciones locales que habían jugado tradicionalmente el papel de colchón preventivo o, en todo caso, paliativo de los efectos de los propios desastres naturales en cuanto a la reducción de la mortalidad o a la rápida reconstrucción de las comunidades que los padecían, dejándolas a la intemperie y abocándolas a esa situación de desamparo, de vulnerabilidad material y social cronificada, que constituye lo que desde hace décadas venimos conociendo como el Tercer Mundo.

2. El propio Amartya Sen ha colaborado con organismos internacionales elaborando informes sobre desarrollo humano. A. Sen y M. Nussbaum trabajaron conjuntamente en el Informe sobre desarrollo humano del Programa de las Naciones Unidas para el desarrollo (PNUD), elaborando una propuesta de análisis de las capacidades humanas como clave del desarrollo. Definieron una serie de dimensiones esenciales de la vida humana cuya carencia implica que no puede llamársele digna, de manera que cualquier política debe tener como meta el desarrollo y preservación de dichas capacidades. Esta perspectiva sobre la vulnerabilidad ha alimentado numerosos modelos teóricos, muchos de ellos formulados al amparo de los estudios de desarrollo financiados por organismos internacionales, entre otros: el modelo PSR (Pressure, State, Response) desarrollado por la OCDE, un estudio de referencia sobre la vulnerabilidad ambiental, la perspectiva SRM (Social Risk Management) del Banco Mundial y el enfoque AVEO (Activos-Vulnerabilidad-Estructura de Oportunidades) de la CEPAL.

3. En este artículo, Sirven se propone trazar las principales evoluciones conceptuales y metodológicas del análisis de la pobreza en las últimas décadas hasta concebir los útiles susceptibles de anticipar la pobreza, en particular a través de la noción de vulnerabilidad. Él aprecia, no una ruptura, sino más bien una continuidad entre las perspectivas ex post (pobreza) y ex ante (vulnerabilidad).

4. En este apartado he seguido muy de cerca la exposición que hace Moreno Crossley de esos dos enfoques de la vulnerabilidad social, pero he intercambiado el orden que él propone para su examen (él presenta en primer lugar la concepción de vulnerabilidad y fragilidad y en segundo lugar la concepción de vulnerabilidad y riesgo), porque los textos de la que él considera primera en realidad son posteriores. Dado que este artículo se propone trazar una genealogía, no parece oportuno referirse a los textos en orden inverso a la fecha de su publicación.

5. Dentro de las concepciones de la vulnerabilidad asociada al riesgo, el autor distingue otros dos grupos de definiciones. La primera, que acabamos de mencionar, tiene que ver con la visión tradicional, o de sentido común, y se ha empleado desde diversas disciplinas porque las situaciones de las que dan cuenta son muy variadas (catástrofes naturales, epidemias, escasez material, anomia, etc.). La segunda, en la que nos hemos detenido, es la característicamente sociológica. La tercera sería la vinculada con la investigación empírica orientada a las políticas públicas. Esta última tiene una vocación más operativa, por lo que su contribución se ha centrado, más que en desarrollar aspectos conceptuales, en la construcción de indicadores de vulnerabilidad que permitan el diseño y la aplicación de políticas sociales.

6. Basamos nuestra exposición en el texto Activos y estructuras de oportunidades. Estudios sobre las 
raíces de la vulnerabilidad social en Uruguay, publicado por la oficina de la CEPAL en Montevideo (1999), que contiene la presentación más completa del enfoque teórico-metodológico AVEO. Aunque este texto contiene tanto el desarrollo conceptual como el desarrollo metodológico de este enfoque, nos centraremos únicamente en delinear el marco conceptual, que es el que nos interesa a efectos de analizar el concepto de vulnerabilidad que maneja.

7. Nosotros nos hemos detenido particularmente en el enfoque AVEO, pero alrededor de los 2000 y en la primera década del XXI hubo una proliferación considerable de trabajos sobre vulnerabilidad social realizados por especialistas latinoamericanos.

8. Jorge Grau ha hecho el ejercicio de revisar las métricas de publicación científica en los últimos 20 años rastreando los estudios sobre vulnerabilidad con el siguiente resultado: "Si tomamos a Scopus como botón de muestra, en el conjunto de disciplinas científicas con publicaciones indexadas en su repositorio el término 'vulnerability' aparece en títulos, resúmenes o palabras clave un total de 19283 veces en el decenio 1997-2007, elevándose esa cifra a 66305 en el período 2008-2020, lo que supone un incremento del 243,85\%" (Grau y otros 2021, en prensa).

\section{Bibliografía}

Beck, Ulrich

1986 La sociedad del riesgo: hacia una nueva modernidad. Barcelona, Paidós, 1998.

Davis, Mikes

2001 Los holocaustos de la era victoriana. El Niño, las hambrunas y la formación del Tercer Mundo. Valencia, Publicacions de la Universitat de València, 2006.

Feito, Lydia

2007 "Vulnerabilidad", Anales del Sistema Sanitario de Navarra, n 30, supl. 3: 7-22.

Filgueira, Carlos

2001 La actualidad de viejas temáticas: sobre los estudios de clases, estratificación y movilidad social en América Latina. Santiago, CEPAL.

González, Leandro M.

2009 "Orientaciones de lectura sobre vulnerabilidad social”, en Leandro M. González (comp.), Lecturas sobre vulnerabilidad y desigualdad social. Córdoba, Centro de Estudios Avanzados CONICET: 13-29.

Grau, Jorge (y otros)

2021 "Vulnerabilidad sociocultural en contextos de crianza: retos teóricos y propuesta analítica", Disparidades. Revista de Antropología (en prensa).

Katzman, Rubén (y otros)

1999 Activos y estructuras de oportunidades. Estudios sobre las raíces de la vulnerabilidad social en Uruguay. Montevideo, CEPAL.

Moreno, Juan Cristóbal

2008 "El concepto de vulnerabilidad social en el debate en torno a la desigualdad: problemas, alcances y perspectivas", Observatory on Structures and Institutions of Inequality in Latin America, Working Paper Series, $n^{\circ}$ 9, Center for Latin American Studies, University of Miami: 3-38.

Moser, Caroline 1998 "Reassessing urban poverty reduction strategies: The asset vulnerability framework", World Development, Washington, The World Bank, vol. 26, $\mathrm{n}^{\circ}$ 1: 1-19. 


\section{Pérez, Karlos}

2000 "Vulnerabilidad", Diccionario de acción humanitaria y cooperación al desarrollo, Universidad del País Vasco: 1-13.

Sen, Amartya

1981 Poverty and Famines. Oxford, Clarendon Press.

Sirven, Nicolas

2007 "De la pauvreté à la vulnérabilité: évolutions conceptuelles et méthodologiques", Mondes en développement, $\mathrm{n}^{\circ}$ 140: 9-24. 